
and Thrombosis

\title{
Failure of Platelet Parameters and Biomarkers to Correlate Platelet Function to Severity and Etiology of Heart Failure in Patients Enrolled in the EPCOT Trial
}

\author{
With Special Reference to the Hemodyne ${ }^{\circledR}$ Hemostatic Analyzer
}

\author{
Victor L. Serebruany ${ }^{a}$ Marcus E. McKenzie ${ }^{a}$ Andrew F. Meister ${ }^{a}$ \\ Sergey Y. Fuzaylova Paul A. Gurbel ${ }^{a}$ Dan Atarb Wendy A. Gattis ${ }^{c}$ \\ Christopher M. O'Connorc
}

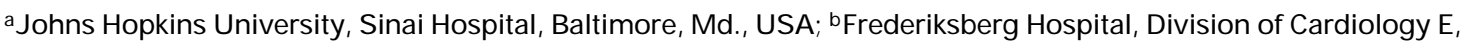

Copenhagen, Denmark; ' Duke Clinical Research I, Durham, N.C., USA

\section{Key Words \\ Aggregation - Congestive heart failure - Glycoprotein Ilb/Illa $\cdot$ Human Platelet function - Selectin . \\ Thrombocyte contractile force}

\begin{abstract}
Data from small studies have suggested the presence of platelet abnormalities in patients with congestive heart failure (CHF). We sought to characterize the diagnostic utility of different platelet parameters and platelet-endothelial biomarkers in a random outpatient CHF population investigated in the EPCOT ('Whole Blood Impedance Aggregometry for the Assessment of Platelet Function in Patients with Congestive Heart Failure') Trial. Blood samples were obtained for measurement of platelet contractile force (PCF), whole blood aggregation, shear-induced closure time, expression of glycoprotein (GP) Ilb/llla, and P-selectin in 100 consecutive patients with CHF. Substantial interindividual variability of platelet characteristics exists in patients with CHF. There were no statistically significant differences when patients were grouped according to incidence of vascular events, emergency re-
\end{abstract}

vascularization needs, survival, or etiology of heart failure. Aspirin use did not affect instrument readings either. PCF correlates very poorly with whole blood aggregometry $\left(r^{2}=0.023\right)$, closure time $\left(r^{2}=0.028\right)$, platelet GP IIb/ Illa $\left(r^{2}=0.0028\right)$, and P-selectin $\left(r^{2}=0.002\right)$ expression. Furthermore, there was no correlation with brain natriuretic peptide concentrations, a marker of severity and prognosis in heart failure reflecting the neurohumoral status. Patients with heart failure enrolled in the EPCOT Trial exhibited a marginal, sometimes oppositely directed change in platelet function, challenging the diagnostic utility of these platelet parameters and biomarkers to serve as useful tools for the identification of platelet abnormalities, for predicting clinical outcomes, or for monitoring antiplatelet strategies in this population. The usefulness of these measurements for assessing platelets in the different clinical settings remains to be explored. Taken together, opposite to our expectations, major clinical characteristics of heart failure did not correlate well with the platelet characteristics investigated in this study.

\begin{tabular}{ll}
\hline KARGER & ( 2002 S. Karger AG, Basel \\
Fax +4161306 12 34 & 1424-8832/02/0321-0008\$18.50/0 \\
$\begin{array}{l}\text { E-Mail karger@karger.ch } \\
\text { www.karger.com }\end{array}$ & $\begin{array}{l}\text { Accessible online at: } \\
\text { www. karger.com/journals/pht }\end{array}$
\end{tabular}

Dr. Victor L. Serebruany

Center for Thrombosis Research, Sinai Hospital of Baltimore

2401 West Belvedere Avenue, Schapiro Research Building R-202

Baltimore, MD 21215 (USA)

Tel. +1 410601 5266, Fax +1 410601 9061, E-Mail heartdrug@aol.com 


\section{Introduction}

Congestive heart failure (CHF) is a major health problem among Americans, with an estimated prevalence of 4-5 million [1]. Despite major advances in treatment, including ACE inhibitors and $\beta$-blockers, mortality remains high [2]. In recent years, evidence has been presented showing that patients who were receiving anticoagulant therapy in the setting of CHF had lower mortality rates than their non-anticoagulated counterparts [3]. The Studies of Left Ventricular Dysfunction Trial showed significantly reduced numbers of both cardiovascular-related deaths as well as overall hospital admissions for patients with CHF on warfarin [4]. As early as in the 1970s, studies have suggested that platelet activity is increased in patients with CHF [5]. Markers of platelet activation have been shown to be increased in these patients, including $\beta$-thromboglobulin [6-8], P-selectin [9], PECAM-1 [10], and osteonectin [10]. Markers of thrombin activation and fibrinolysis, such as fibrinopeptide A, D-dimer, and thrombin-anti-thrombin III complexes, are also elevated in CHF [6, 7, 11]. Interestingly, studies have shown an elevation in several of the above markers regardless of whether the CHF was ischemic or non-ischemic in etiology $[6,7,11]$. The exact role of a possible procoagulant state in the development and progression of CHF is unknown at this time, and studies are ongoing.

Platelet activation may play an important role in the deterioration of the failing heart due to the development of microthrombi in the myocardium. Conventional platelet-rich plasma aggregometry is still the gold standard for the majority of clinical laboratories assessing platelet function. However, new screening devices are rapidly evolving. For example, an instrument recently developed allows simultaneous monitoring of platelet function and clot structure (in clots formed from platelet-rich plasma or whole blood), in that platelet function is assessed by measuring the force developed by platelets during clot retraction. Clot structure is observed by the development of elasticity during clot formation. Other assessments of potential significance are the so-called platelet closure time utilizing a particular analyzer, whole blood impedance aggregometry using an aggregometer, or the measurements of biomarkers such as glycoprotein (GP) IIb/ IIIa or P-selectin.

We sought to determine the diagnostic utility of these parameters and biomarkers with the goal to identify patients with heightened platelet activity in the CHF population. Further we attempted to correlate platelet con-
Table 1. Clinical characteristics of the $\mathrm{CHF}$ patients

\begin{tabular}{ll}
\hline Parameter & Patients \\
\hline Demographics & \\
$\quad$ Age, years & $65(53,73)$ \\
$\quad$ Male gender, \% & 71 \\
Caucasians, \% & 64 \\
$\quad$ African-American, \% & 32 \\
NYHA class & \\
I & 1 \\
II & 41 \\
III & 46 \\
IV & 12 \\
Median LVEF & $20(15,30)$ \\
Ischemic etiology, \% & 61 \\
Hypertensive etiology, \% & 18 \\
Cardiomyopathy, \% & 9 \\
End-stage valvular disease, \% & 7 \\
Alcohol-induced CMP & 5 \\
Medications, \% & \\
$\quad$ B-Blocker & 66 \\
ACE inhibitor & 78 \\
Warfarin & 17 \\
Diuretics & 44 \\
Aspirin & 63 \\
\hline CMP = Cardiomyopathy; LVEF = left \\
ventricular ejection fraction. Numbers in pa- \\
rentheses = 25 and 75 percentile range. \\
L
\end{tabular}

tractile force (PCF) measurements in blood samples from CHF patients to closure time using the Platelet Function Analyzer (PFA-100 ${ }^{\circledR}$ ), impedance whole blood aggregometry, expression of GP IIb/IIIa and P-selectin expression by whole blood flow cytometry, as well as brain natriuretic peptide (BNP), a recently discovered marker of severity and prognosis in heart failure patients reflecting the neurohumoral status.

\section{Patients and Methods}

Patients

The regional ethics committee approved the study, and all subjects gave informed consent. One hundred consecutive outpatients were enrolled at the Duke University Heart Failure Clinic and investigated in the EPCOT ('Whole Blood Impedance Aggregometry for the Assessment of Platelet Function in Patients with Congestive Heart Failure') Trial. Detailed patients' clinical characteristics are presented in table 1. All had symptoms for at least 3 months, with no change in medication during the last month. Information on antecedent aspirin use was obtained as part of the study protocol. Aspirin use 
Table 2. Correlation of clinical characteristics of $\mathrm{CHF}$ patients and platelet measures

\begin{tabular}{llllllllllll}
\hline Parameter & $\mathrm{n}$ & $\begin{array}{l}\text { PCF } \\
\text { kdyn }\end{array}$ & $\mathrm{p}$ & $\begin{array}{l}\text { WBA } \\
\Omega\end{array}$ & $\mathrm{p}$ & $\begin{array}{l}\text { PFA-100 } \\
\mathrm{s}\end{array}$ & $\mathrm{p}$ & $\begin{array}{l}\text { GP IIb/IIIa } \\
\text { log MFI }\end{array}$ & $\mathrm{p}$ & $\begin{array}{l}\text { P-selectin } \\
\% \text { increase }\end{array}$ \\
\hline Clinical event* & 39 & $13(10,20)$ & 0.94 & $25(22,30)$ & 0.55 & $91(69,116)$ & 0.63 & $577(513,674)$ & 0.89 & $12(7,15)$ & 0.22 \\
No clinical event & 53 & $14(9,18)$ & & $24(21,27)$ & & $90(80,116)$ & & $587(539,623)$ & & $10(6,13)$ & \\
Ischemic etiology & 59 & $15(11,20)$ & 0.77 & $27(23,31)$ & 0.09 & $93(67,116)$ & 0.37 & $594(498,657)$ & 0.83 & $13(6,18)$ & 0.76 \\
Non-ischemic etiology & 33 & $14(10,18)$ & & $22(17,25)$ & & $94(67,119)$ & & $570(468,636)$ & & $14(8,17)$ & \\
NYHA class I-II & 40 & $13(9,17)$ & 0.40 & $24(21,26)$ & 0.26 & $92(76,122)$ & 0.42 & $578(517,716)$ & 0.28 & $14(8,16)$ & 0.15 \\
NYHA class III-IV & 52 & $13(10,19)$ & & $24(22,29)$ & & $88(73,112)$ & & $586(531,681)$ & & $11(6,14)$ & \\
LVEF<20\% & 51 & $13(9,19)$ & 0.78 & $24(22,28)$ & 0.91 & $86(74,104)$ & 0.08 & $583(493,623)$ & 0.24 & $10(5,14)$ & 0.17 \\
LVEF > 20\% & 41 & $14(10,18)$ & & $25(21,28)$ & & $97(77,130)$ & & $595(542,673)$ & & $13(7,16)$ & \\
Aspirin & 60 & $13(9,17)$ & 0.69 & $22(16,26)$ & 0.24 & $103(89,121)$ & 0.10 & $572(485,629)$ & 0.65 & $12(7,16)$ & 0.21 \\
No aspirin & 32 & $15(10,19)$ & & $25(19,29)$ & & $88(67,109)$ & & $597(468,647)$ & & $15(9,18)$ & \\
\hline
\end{tabular}

* Clinical events include emergency revascularization, acute myocardial infarction, stroke, or death. Numbers in parentheses $=25$ and 75 percentile range. $\mathrm{LVEF}=$ Left ventricular ejection fraction.

was defined as self-reporting daily consumption of at least $81 \mathrm{mg}$ of acetylsalicylic-acid-containing medication (coated or uncoated). Patients with decompensated heart failure, or receiving an antithrombotic agent other than aspirin or warfarin, were excluded.

\section{Samples}

Blood was drawn between 8 and 10 a.m. in order to avoid any diurnal influence and sampled from an antecubital vein using a 19gauge butterfly needle into a tube containing $3.8 \%$ sodium citrate (1:9 volume) after having discarded the first $1.5 \mathrm{ml}$ of free running blood. Three Vacutainer tubes $(7.0 \mathrm{ml})$ were collected for a total of 21 $\mathrm{ml}$ of whole blood-citrate mixture from each patient. To avoid possible observer bias, blood samples were coded and blinded. Individuals unaware of the purpose of the study performed sampling procedures and platelet studies. PCF by the RM2 Instrument (Hemodyne, Richmond, Va., USA), whole blood aggregometry (model 591/592, Chronolog, Havertown, Pa., USA), as well as closure time by PFA100 (Dade Behring, Miami, Fla., USA), whole blood flow cytometry (FACScan, Becton Dickinson, San Diego, Calif., USA) and BNP (Elecsys; Roche, Basle, Switzerland) were measured.

\section{Platelet Contractile Force}

The operating procedures for the Hemostatic Analyzer are described in detail elsewhere [12]. The apparatus is measuring the force developed by platelets as they undergo cellular contraction, and speed of clot formation in whole blood between a thermostated cup and parallel upper plate. Before gelation, the upper plate is centered above the cup and lowered into the clotting solution. As the clot forms, it attaches to the inner walls of the cup and the bottom of the upper plate. A small portion of the upper clot surface between the outer edge of the upper plate and the inner wall of the lower cup is initially exposed to air. Covering the air-clot interface with a thin layer of silicone oil prevents evaporation. Once clotting is complete, platelets within the network pull fibrin strands inwards thereby transmitting force through the network to the surfaces the clot adheres to. Force measurement is accomplished utilizing a force displacement transducer coupled to the upper plate, and is expressed in kilodynes [13].

\section{Whole Blood Impedance Platelet Aggregation}

The Chrono-log Whole Blood Impedance Aggregometer (model 591/592) is a simple, compact instrument suitable for routine determinations of platelet aggregation in whole blood. The method is described in details elsewhere [14]. Briefly, a dilution 1:1 with $0.5 \mathrm{ml}$ TBS specimen of whole blood is made in the sample cuvette which is placed in the device reaction well. The electrode is inserted in the sample cuvette, and the platelets in the blood sample adhere to two fine palladium wires on the electrode, forming uniform monolayers of platelets coating the wires. A small voltage difference is applied across the two wires, and the impedance caused by the platelets coating the wires is measured. In the absence of an aggregating agent or agonist, the interactions between the platelets and the electrodes stabilize, and the impedance between the two electrodes becomes constant, producing a baseline. When an agonist is added, platelets in the specimen are activated and begin to aggregate. The coating of platelets thickens on the wires over the next several minutes with a corresponding increase in electrical impedance between the electrode wires. This change in impedance is directly proportional to the extent of platelet aggregation and is indicated on the digital display in ohms after $6 \mathrm{~min}$. Platelet aggregation was stimulated with $20 \mu \mathrm{mol}$ ADP. Platelet aggregability was expressed as the change in electrical impedance and is expressed in ohms. Aggregation curves were recorded for $6 \mathrm{~min}$ and analyzed using the Aggrolink ${ }^{\circledR}$ software.

\section{Closure Time}

The PFA- $100^{\circledR}$ is a high-shear-inducing analyzer that simulates primary hemostasis after injury to a small vessel under flow conditions [15]. The device provides a constant negative pressure that aspirates a whole blood citrate mixture that comes into contact with a collagen-coated membrane and then passes through an aperture. The time required to obtain occlusion of the aperture is digitally recorded. Precision testing is reported to show a coefficient of variation of $<10 \%$ for within-day and between-day analyses on collagen/ADP cartridges and 5-14\% for collagen/epinephrine cartridges [16]. Collagen/epinephrine cartridges are reported to show qualitative platelet defects, such as acetylsalicylic-acid-induced abnormalities, while collagen/ADP cartridges show thrombocytopathies but not qualitative platelet abnormalities. 


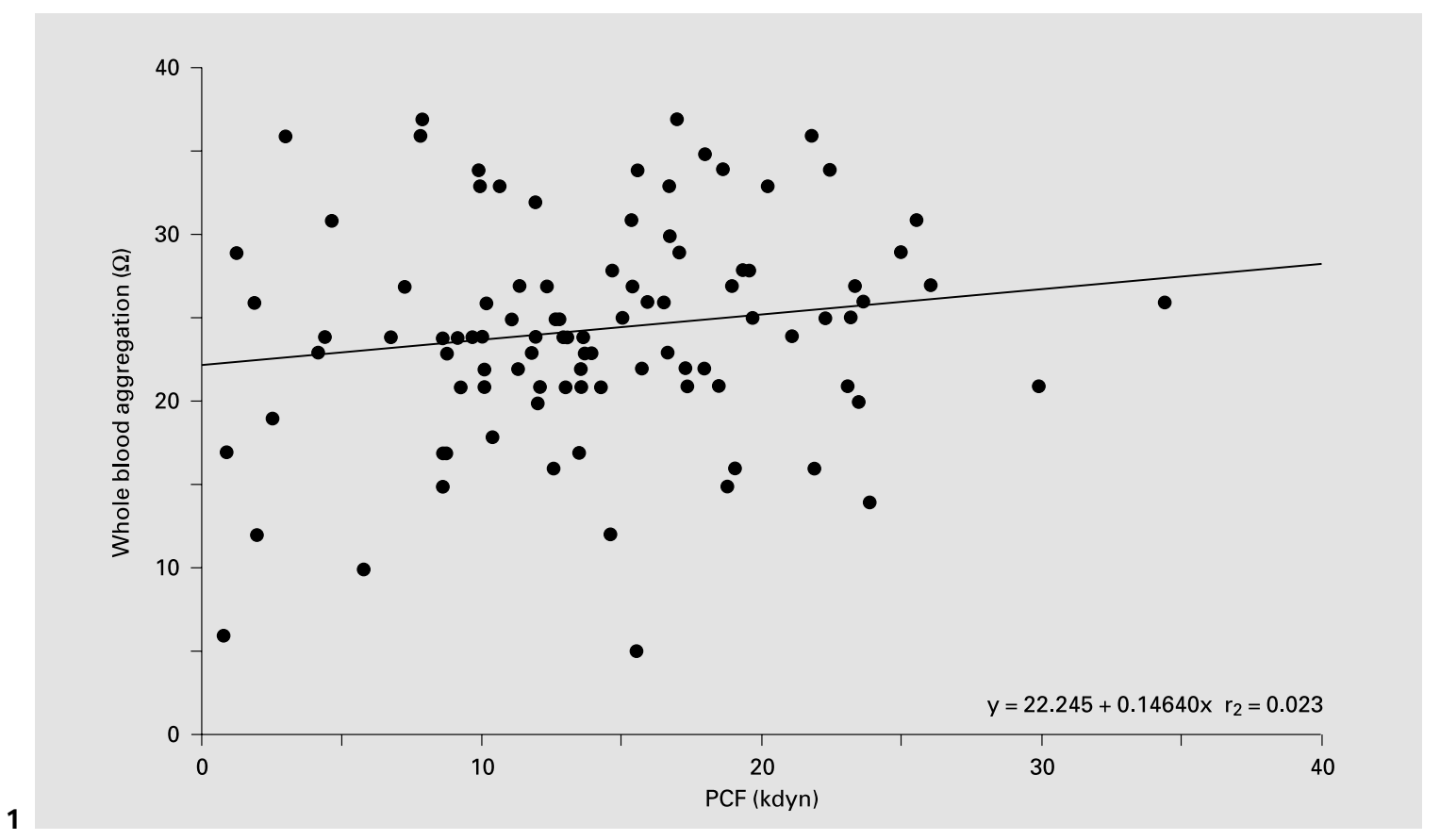

Fig. 1-5. Graphs illustrate the correlation between PCF with whole blood impedance platelet aggregability induced by $20 \mu M$ ADP (1), shear-induced closure time (2), platelet expression of GP IIb/IIIa (3), P-selectin (4), and BNP (5). $\mathrm{r}^{2}>0.3$ is usually considered significant in regression analysis.

\section{Whole Blood Flow Cytometry}

The method has been described in detail previously [17, 18]. TBS buffer and fluorescein-isothiocyanate-conjugated monoclonal antibodies to P-selectin (PharMingen, San Diego, Calif., USA) and GP IIb/IIIa (DAKO, Carpenteria, Calif., USA) were removed from the refrigerator and allowed to warm at room temperature. Amber tubes $(1.25 \mathrm{ml})$ and Eppendorf tubes $(1.5 \mathrm{ml})$ were marked and labeled appropriately. Then $450 \mu \mathrm{l}$ of TBS buffer was pipetted into an Eppendorf tube, mixed with $50 \mu \mathrm{l}$ of whole blood, and incubated with $5 \mu \mathrm{l}$ of appropriate antibody at room temperature for $30 \mathrm{~min}$. After incubation, $400 \mu \mathrm{l}$ of $2 \%$ buffered paraformaldehyde was added. Samples were stored in the refrigerator at $4^{\circ} \mathrm{C}$ until further analysis within $48 \mathrm{~h}$ using a FACScan flow cytometer. The data were collected in list mode files and then analyzed using the CellQuest ${ }^{\circledR}(1.3)$ software.

\section{Statistical Analyses}

A simple linear regression analysis was performed to identify the relation between the different platelet measures in the same patient. An $\mathrm{r}^{2}>0.300$ is usually considered significant in regression analyses.

\section{Results}

Clinical characteristics of the patients are presented in table 1 . In terms of age distribution (average 65 years), impaired left ventricular function (median LV ejection fraction 20\%), NYHA class (class II $41 \%$ and class III $46 \%$, respectively), and etiology (61\% ischemic), the cohort is highly representative of a typical CHF population. Furthermore, the table depicts the use of state-of-the-art anticongestive medication.

Table 2 shows how clinical characteristics correlate with PCF, aggregometry, platelet closure time, GP IIb/ IIIa expression, or P-selectin measurement. None of the subgroups showed significant correlation to these parameters.

For the final analysis of the correlation between the various platelet parameters, 8 samples had to be excluded due to technical quality control concerns. The remaining 92 samples were analyzed and displayed in figures 1-5. These graphs represent individual plots of how well PCF correlates with whole blood aggregation (fig. 1), shearinduced closure time (fig. 2), expression of GP IIb/IIIa (fig. 3), platelet P-selectin (fig. 4) and BNP (fig. 5). In conclusion, PCF correlates very poorly with whole blood aggregometry $\left(\mathrm{r}^{2}=0.023\right)$, closure time $\left(\mathrm{r}^{2}=0.028\right)$, platelet GP IIb/IIIa $\left(r^{2}=0.0028\right)$, P-selectin $\left(r^{2}=0.002\right)$ and $\operatorname{BNP}\left(r^{2}=0.001\right)$.

Pathophysiol Haemost Thromb 2002;32:8-15 

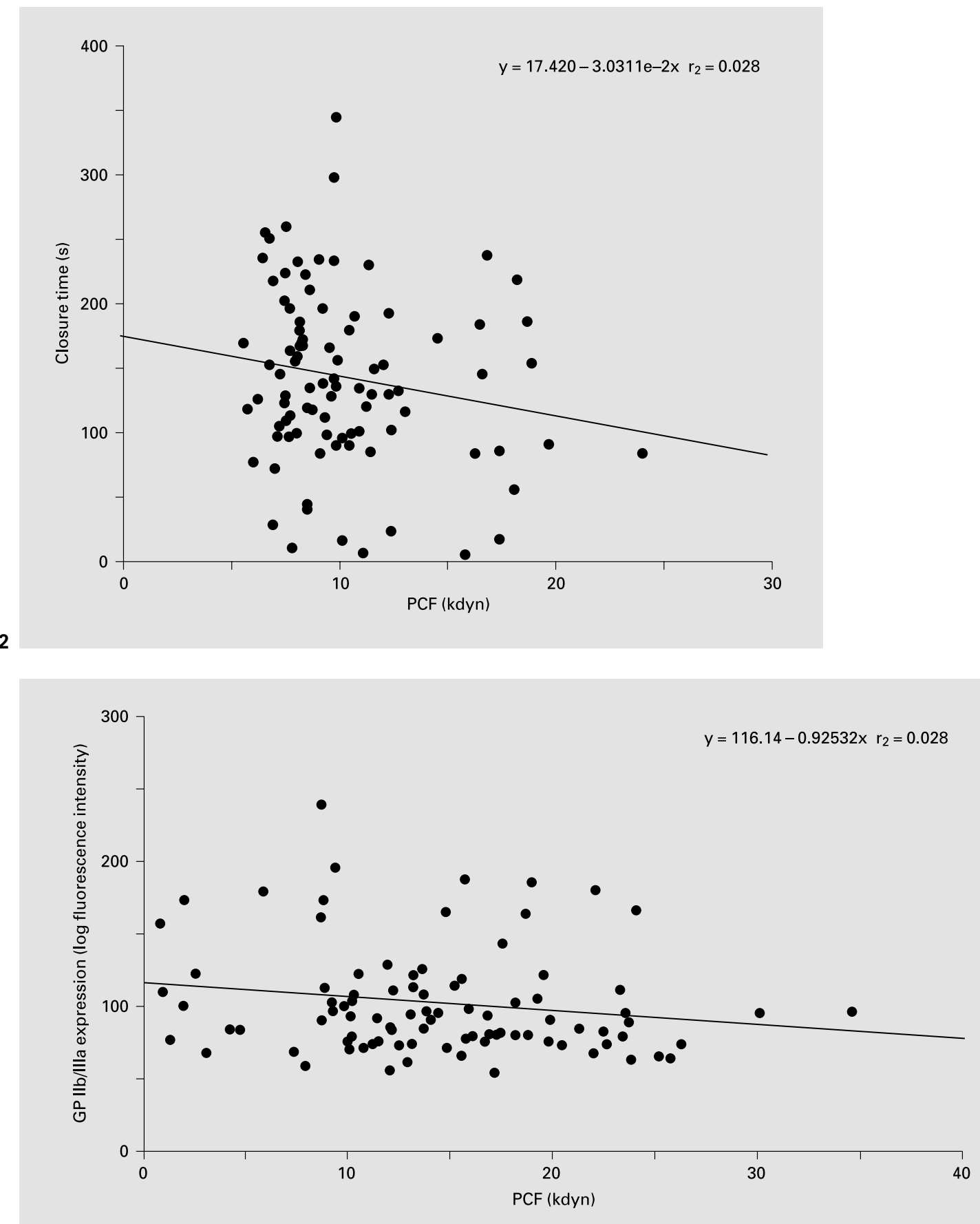

3

\section{Discussion}

The important role of platelets in patients with CHF has received increasing recognition in recent years. One attractive theory links CHF with acquired platelet dysfunction. Most researchers agree that platelet-related characteristics could affect both short- and long-term outcome in patients with CHF. Platelet activity is not currently measured in patients with $\mathrm{CHF}$ as a matter of routine. However, as more is learned about the role of heightened platelet function, and as initial attempts have been made to introduce antiplatelet therapy in this population, 

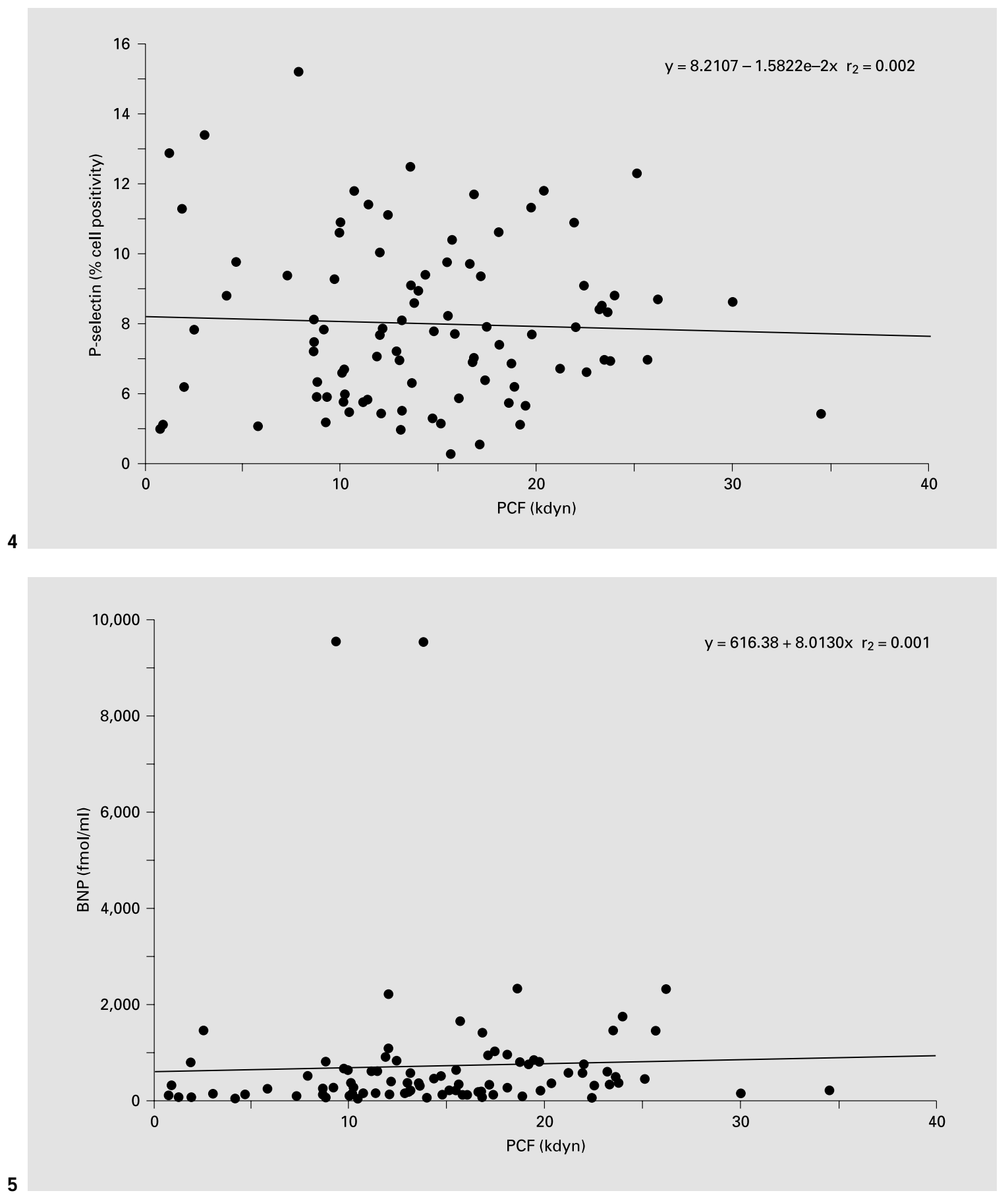

the ability to quantify the platelet status becomes more clinically relevant.

The Hemodyne Hemostatic Analyzer (model RM2) is a relatively new instrument based on an original idea for measuring the forces generated by stimulated platelets during clot retraction [12]. Utilizing this technique, de- creased platelet force development may for example be detected in uremic patients with qualitative platelet dysfunction [19], and enhanced force development has been reported in patients with severe coronary artery disease [20]. 
There are limited data supporting measurement of PCF as a valuable tool to detect patients with platelet dysfunction more efficiently and cost-effectively than conventional aggregometry, and supporting a potential use for monitoring the efficacy of antiplatelet agents including GP IIb/IIIa inhibitors [21]. The diagnostic utility of the Hemodyne Analyzer has been suggested for use in assessing the function of stored platelet concentrates and platelets that have undergone freezing or exposure to alternative buffers [22], which is relevant to blood bank operations. Although this technology has been shown to provide interesting data in a research setting, the ability of the Hemodyne Hemostatic Analyzer to detect platelet abnormalities in a clinical laboratory setting remains to be established [23].

Surprisingly, we have found no correlation whatsoever of the PCF with any of the previously established means used in platelet function assessment (WBA, closure time, GP IIb/IIIa and P-selectin expression) suggesting that measurement of PCF is not a valuable method for the assessment of platelet function, at least in the $\mathrm{CHF}$ patients. There were no differences in the PCF dependent on the clinical characteristics of heart failure or aspirin use. On the other hand, there were no significant differences either in the other four parameters assessed (aggregometry, closure time, GP IIa/IIIb, or P-selectin) towards severity or etiology of heart failure. There may be several explanations to these facts. First, platelet function may not be necessarily directly related to the severity of heart failure. It is entirely unknown whether platelet activity is associated with the outcome, prognosis, and deterioration of the failing myocardium. Second, considering that $\mathrm{CHF}$ patients are under treatment with many medications (a 5.6 drugs average for each EPCOT participant), other pharmaceuticals may interfere with aspirin, reducing, or at least compromising the antiplatelet effect. Finally, there is a variety of other confounding factors (e.g. biomarkers and hormone disbalance, or coagulopathy) jeopardizing our ability to detect minor platelet abnormalities in these patients. In short, the clinical setting of heart failure is not an ideal 'drawing board' for testing novel devices assessing platelets.

It is very difficult to explain why PCF does not correlate at all with other conventional platelet tests. It is possible, but highly unlikely, that platelet contractility is unrelated to aggregation and/or receptor expression. Moreover, stimulation of platelets with thrombin, which is used in the Hemodyne protocol to enhance PCF, may result in an immediate clot formation. It remains to be elucidated if we are truly measuring platelet rather than clot contractile force and whether or not this particular instrument is indeed a platelet function analyzer.

In order to shed more light on the neurohumoral status of our heart failure cohort, we attempted to correlate PCF to BNP, as this marker has been shown to both reflect severity of heart failure and prognosis. Again there was no correlation to be found between these two parameters.

In conclusion, patients with heart failure enrolled in the EPCOT Trial exhibited marginal, sometimes oppositely directed, changes in the platelet function, challenging the diagnostic utility of platelet parameters and biomarkers to serve as a useful tool for the identification of platelet abnormalities, or for monitoring antiplatelet strategies in this population. Interestingly, this finding is reminiscent of heterogeneous changes in platelet functions observed after thrombolysis [24]. Particularly, the usefulness of the Hemodyne Platelet Analyzer for assessing platelets in the different clinical CHF settings remains to be explored. Taken together, and opposite to our expectations, major clinical characteristics of heart failure did not correlate well with the platelet characteristics investigated here.

\section{Acknowledgments}

This work was supported by HeartDrug ${ }^{\mathrm{TM}}$ Research, LLC (Wilmington, Del., USA), the Duke Clinical Research Institute (Durham, N.C., USA), and the Danish Heart Foundation (grant No. 00-2-346-22854 to D.A.). Part of this study was presented as an abstract at the XXIInd Congress of the European Society of Cardiology (Amsterdam, The Netherlands; August 26-30, 2000). 


\section{References}

1 Willimas JF Jr, Bristow MR, Fowler MB, Francis GR, Garson A, Gersh BJ, Hammer DF, Hiatky MA, Leier CV, Packer M, Pitt B, Ullyot DJ, Wexler LF, Winters WL: Guidelines for the evaluation and management of heart failure. Report of the American College of Cardiology/American Heart Association Task Force on Practice Guidelines (Committee on Evaluation and Management of Heart Failure). J Am Coll Cardiol 1995;26:1376-1398.

2 Ho KK, Anderson KM, Kannel WB, Grossman W, Levy D: Survival after the onset of congestive heart failure in Framingham Heart Study subjects. Circulation 1993;88:107-115.

3 Al-Khadra AS, Salem DN, Rand WM, Udelson JE, Smith JJ, Konstam MA: Warfarin anticoagulation and survival: A cohort analysis from the Studies of Left Ventricular Dysfunction (SOLVD) Trial. J Am Coll Cardiol 1998;31: 749-753.

4 Al-Khadra AS, Salem DN, Rand WM, Udelson JE, Smith JJ, Konstam MA: Antiplatelet agents and survival: A cohort analysis from the Studies of Left Ventricular Dysfunction (SOLVD) Trial. J Am Coll Cardiol 1998;31:419-425.

5 Mehta J, Mehta P: Platelet function studies in heart disease. VI. Enhanced platelet aggregate formation activity in congestive heart failure Inhibition by sodium nitroprusside. Circulation 1979;60:497-503.

6 Jafri SM, Ozawa T, Mammen E, Levine TB, Johnson C, Goldstein S: Platelet function, thrombin and fibrinolytic activity in patients with heart failure. Eur Heart J 1993;14:205212.

7 Sbarouni E, Bradshaw A, Andreotti F, Tuddenham E, Oakley CM, Cleland JG: Relationship between hemostatic abnormalities and neuroendocrine activity in heart failure. Am Heart J 1994;127:607-612.
8 Jafri SM, Riddle JM, Raman SB, Goldstein S: Altered platelet function in patients with severe congestive heart failure. Henry Ford Hosp Med J 1986;34:156-159.

9 O'Connor CM, Gurbel PA, Serebruany VL: Usefulness of soluble and surface-bound Pselectin in detecting heightened platelet activity in patients with congestive heart failure. Am J Cardiol 1999;83:1345-1349.

10 Serebruany VL, Murugesan SR, Pothula A, Atar D, Lowry D, Gattis WA, O'Connor CM, Gurbel PA: Increased soluble platelet/endothelial cellular adhesion molecule-1 and osteonectin levels in patients with severe congestive heart failure. Independence of disease etiology, and antecedent aspirin therapy. Eur J Heart Fail 1999;1:243-249.

11 Yamamoto K, Ikeda U, Furuhashi K, Irokawa M, Nakayama T, Shimada K: The coagulation system is activated in idiopathic cardiomyopathy. J Am Coll Cardiol 1995;25:16341640.

12 Carr ME, Zekert SL: Measurement of plateletmediated force development during plasma clot formation. Am J Med Sci 1991;302:1318 .

13 Greilich PE, Carr ME, Carr SL, Chang AS: Reductions in platelet force development by cardiopulmonary bypass are associated with hemorrhage. Anesth Analg 1995;80:459-465.

14 Cardinal DC, Flower RJ: The electric aggregometer: A novel device for assessing platelet behavior in blood. J Pharmacol Methods 1980; 3:135-158.

15 Kundu SK, Heilman EJ, Sio R, Garcia C, Ostgaard RA: Characterization of an in vitro platelet function analyzer, PFA- $100^{\circledR}$. Clin Appl Thromb Hemost 1996;2:241-249.
16 Mammen EF, Alshameeri RS, Comp PC: Preliminary data from the field trial of the PFA100 system. Semin Thromb Hemost 1995; 21(suppl 2):113-121.

17 Ault KA: Flow cytometric measurement of platelet function and reticulated platelets. Ann NY Acad Sci 1993;677:293-308.

18 Serebruany VL, Kereiakes DJ, Dalesandro MR, Gurbel PA: Model of flow cytometer markedly affects platelet-bound P-selectin expression in patients with chest pain. Are we comparing apples with oranges? Thromb Res 1999;96:51-56.

19 Carr ME, Zekert SL: Force monitoring of clot retraction during DDAVP therapy for the qualitative platelet disorder of uraemia: Report of a case. Blood Coagul Fibrinolysis 1991;2:303307.

20 Greilich P, Carr ME, Zekert SL: Effects of aspirin on clot structure and fibrin platelet interactions in patients with severe coronary artery disease. Clin Res 1992;40:241A.

21 Greilich PE, Alving BM, Longnecker D, Carr ME Jr, Whitten CW, Chang AS, Reid TJ: Nearsite monitoring of the antiplatelet drug abciximab using the Hemodyne analyzer and modified thrombelastograph. J Cardiothorac Vasc Anesth 1999;13:58-64.

22 Reid TJ, Snider R, Hartman K, Greilich PE, Carr ME, Alving BM: A method for the quantitative assessment of platelet-induced clot retraction and clot strength in fresh and stored platelets. Vox Sang 1998;75:270-277.

23 Carr ME Jr: In vitro assessment of platelet function. Transfus Med Rev 1997;11:106115.

24 Callahan KP, Malinin AI, Gurbel PA, Alexander JH, Granger CB, Atar D, Serebruany VL: Platelets and thrombolysis: Cooperation or contrariety? Heart Drug 2001;1:281-290. 